\title{
Influence of gamma-ray irradiation on optical and thermal degradation of poly(ethyl-methacrylate) (PEMA) polymer
}

\section{S. Fares}

National Center for Radiation Research and Technology (NCRRT), Nasr City, Egypt; sfares2@yahoo.com

Received 23 April 2012; revised 26 May 2012; accepted 11 June 2012

\begin{abstract}
Polymer based on poly(ethyl-methacrylate) (PEMA) were exposed to different doses of gamma radiation up to $100 \mathrm{kGy}$ and the physical properties have been studied. The effects of gamma irradiation on the optical spectrum of PEMA films have been investigated using spectrophotometric measurements of reflectance and transmittance in the wavelength range $200-1100$ $\mathrm{nm}$. The structure of the sample is analyzed by $X$-ray diffraction technique and is found to be amorphous and partially crystalline. TGA studies revealed that the thermal stability of polyethyl methacrylate, improved after irradiation doses up to $100 \mathrm{kGy}$. On other hand driving absorption coefficient $\alpha(\omega)$, consequently the band tail width $E_{e}$ and optical band gap estimated. This behavior is believed to be associated with the generation of excess of electronic localized states. Also, Optical constants such as refractive index $(n)$, extinction coefficient $(K)$ have been determined using Swanepole method. Optical dispersion parameters and the dispersion parameters, such as $E_{o}$ (single-oscillator energy), $E_{d}$ (dispersive energy) are discussed in terms of the single-oscillator Wemple-DiDomenico model.
\end{abstract}

Keywords: Gamma Radiation; PEMA; X-Ray

Diffraction Technique; Optical Parameters; Dispersion Parameters; TGA Thermogravimetric

\section{INTRODUCTION}

Acrylic and methacrylic polymers have characteristics of brilliance, optical clarity, high transparency, improved mechanical properties, adhesion capability and chemical stability [1]. The photostability of aliphatic acrylic and methacrylic polymers are generally very high. Applica- tions for rigid methacrylate polymer products include signs and glazing substitute for safety glass and to prevent vandalism, optical fibers for light transmission, plastic eyeglass lenses, dentures and dental filling materials, and contact lenses (hard and soft) [2]. Polymethacrylates degrade to lower molecular weight compounds or to the monomers on heating or irradiation with high energy radiation. The decomposition or stability of methacrylate polymers is related to their structures such as type of ester groups molecular weight and its distribution, stereoregularity and copolymer composition. Although data have been reported on the glass transition temperature [3-5] of poly(ethyl methacrylate), its thermal degradation has been studied [6,7]. [8] Malhotra et al. however in their isothermal weight loss studies report that up to $40 \%$ weight loss values, the thermal decomposition of PEMA is closely resembling that of PMMA. Their GPC analysis also shows that the decomposition of PEMA is dominated by depolymerization. The nature of relaxation mechanisms in amorphous PEMA is discussed on the basis of the TSDC and dielectric results [9].

In general, thermal stability of the polymer composites play a crucial role in determining application, because it effects the final properties of the polymer composites such as the upper-limit use temperature and dimensional stability. For the fabrication of advanced composites with better balance in processing and performance, it is very instructive to characterize the thermal decomposition behavior of the polymer composites. In this regard, thermogravimetric analysis (TGA) is in common use to characterize thermal decomposition behavior and the kinetic parameters of thermal decomposition processes, because of its simplicity and useful information about thermal decomposition [10-14]. Consequently, the understanding of the thermal stability and thermal decomposition kinetics of polymer composites make it possible to develop and extend their applications as various industrial fields. At the same time, the need to develop a molecular-level understanding of the thermal degradation of polymers is becoming increasingly important in areas 
of science and technology associated with the hightemperature processing and combustion of these materials.

Studies continue to focus on developing new methods for increasing the radiation resistance of polymeric materials since radiation exposure is a concern in many polymers applications. The smaller the extent of molecular changes due to irradiation, the more radiation resistant the material. Modification of polymers by irradiation, with ionizing radiation in particular, is an expanding field of research and application because of its technological implications. In addition, $\gamma$-irradiation has been proved to be a significant tool [15] for such modifications. It is well known that $\gamma$-irradiation can amend the electrical, optical, thermal properties etc. of the polymeric materials by changing their morphology through various processes of chain accessioning and cross linking [16-18]. The polymer irradiation leads to shift in optical absorption edges, which shows a lowering of the energy-gap. The decrease in energy band-gap infers an increase in conductivity of the irradiated polymers which might be due to the formation of clusters with rings, or due to the formation of conjugated double bonds or quinonic structures [19].

In the present work the affect of different gamma doses (10 to $100 \mathrm{KGy})$ on the optical and thermal decomposition character of PEMA has been studied by UV-Vis spectroscopy and TGA measurements. The affect of irradiation dose on the optical and thermal properties has been discussed in the light of the polymer's track registration sensitivity. The kinetics of decomposition of PEMA has been studied under non-isothermal conditions using thermo-gravimetry to determine the activation energy $\left(E_{a}\right)$. Thermal behavioral analysis of PEMA as an affect of $\gamma$-irradiation have attracted much attention because of their application in optical devices, and has an important study in this direction.

\section{EXPERIMENTAL}

\subsection{Materials}

Poly(ethyl-methacrylate) (PEMA) with a molecular weight of $\sim 280,000 \mathrm{~g} \cdot \mathrm{mol}^{-1}$ was purchased from Scientific Polymer Products, Inc. (Ontario, NY) and used without further purification.

\subsection{Method}

The samples for these works were prepared by employing the solution casting technique. Films of PEMA were prepared by first dissolving 1 - $2 \mathrm{~g}$ of PEMA in approximately $20 \mathrm{~mL}$ of chloroform. The mixtures were stirred for $24 \mathrm{~h}$ using digital magnetic stirrers to ensure that the PEMA powders fully dissolved. After thoroughly dissolved, the sample was cast into Petri dish and let to dry at room temperature for $48 \mathrm{~h}$ to ensure complete evaporation of the chloroform without promoting thermal degradation. The resultant thin PEMA film $(0.5 \mathrm{~mm})$ was carefully removed from the molds for subsequent analysis.

\subsection{Samples Irradiation}

Sample irradiation was carried out using a ${ }^{60} \mathrm{Co}$ gamma source model ISSLEDOVATEL manufactured by Russian irradiator and located at NCRRT, Egypt. The dose rate was $910 \mathrm{kGy} / 100 \mathrm{~min}$ and the temperature during irradiation was about $40^{\circ} \mathrm{C}$. Irradiation doses ranged from 10 to $100 \mathrm{kGy}$ in air.

\subsection{Structural Characterization and Thermal Decomposition}

X-ray diffraction (XRD) patterns were recorded using Shimadzu diffractometer XRD-6000 x-ray diffraction spectrometer with a copper target $(\lambda=1.542 \AA)$ at operating voltage of $40 \mathrm{kV}$ and an electric current of $30 \mathrm{~mA}$. The optical absorbance and transmittance spectra were recorded against wavelengths of $200-1100 \mathrm{~nm}$ using $\mathrm{UV} / \mathrm{vis}$ unikon 360 double beam spectrophotometer. Thermogravimetric analysis (TGA) were performed using Shimadzu-50 instrument (Japan) at heating rate of $10^{\circ} \mathrm{C} / \mathrm{min}$ under flowing nitrogen $(20 \mathrm{~mL} / \mathrm{min})$ from ambient temperature to $500^{\circ} \mathrm{C}$. The primary TGA thermograms were used to determine the kinetic parameters such as activation energy and order of the thermal decomposition reaction.

\section{RESULTS AND DISCUSSION}

\subsection{X-Ray Diffraction Studies}

The X-ray diffraction pattern of (polyethyl-methacrylate) PEMA samples are shown in Figure 1. The patterns revealed that there are no sharp diffraction lines and the broadened background scattering area of PEMA suggests the presence of amorphous nature, which confirms its non-crystalline structure. Consequently, the increase in $\mathrm{X}$-ray intensity may be resulting from grain growth. The particle consists of grains which are intensity interconnected, causing particles to be almost without porosity. This appears as boarding peaks. When PEMA films are irradiated with $\gamma$-rays, a cross-linked product is formed. The product is dimensionally stable and has increased thermal stability and a significant increase in tensile strength. So, it is well known that the interaction of radiation with polymer materials result in the formation of free radicals by dissociation of the excited states or by ion-molecular reactions. 


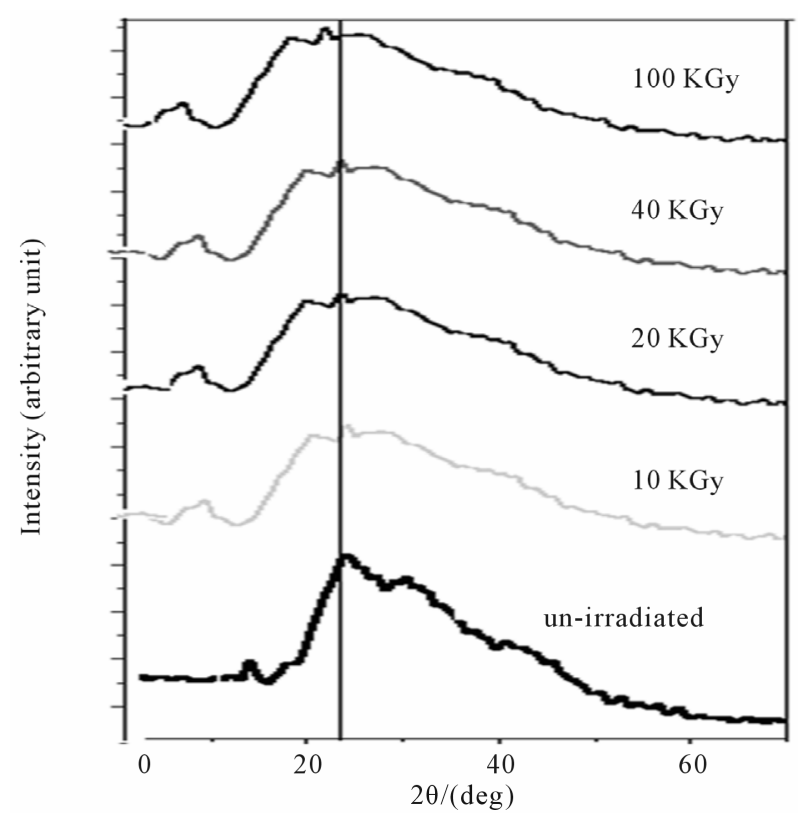

Figure 1. X-ray diffraction patterns of PEMA films before and after irradiation.

\subsection{Thermogravimetric Analysis Techniques (TGA)}

The thermal stability of the polymer composites play a crucial role in determining the limit of their working temperature and the environmental conditions for use, which are related to their thermal decomposition temperature and decomposition rate [20]. To study the affect of $\gamma$-irradiation on these kinetic parameters, TGA thermograms of pristine and gamma irradiated samples of PEMA polymer at different doses have been recorded and are presented in Figure 2. TGA thermograms of PEMA polymer at $100^{\circ} \mathrm{C} / \mathrm{min}$ under nitrogen is shown. TGA curves of PEMA polymer shifted toward higher temperature regions with $\gamma$-irradiation doses, which was explained by the fact that polymer molecules did not have enough time to exhaust the heat with increasing $\gamma$-irradiation doses, leading to slower decomposition rate and higher decomposition temperature due to slow diffusion of heat [21]. TGA provides a method for thermal stability testing. It is observed that there are three stages of decomposition appearing in TGA curves as shown in Figure 2. The first stage at decomposition temperature $\approx$ $2300^{\circ} \mathrm{C}$ is attributed to elimination of evaporated molecules in the side groups. The second stage of weight loss in the temperature range $\approx 230^{\circ} \mathrm{C}-4000^{\circ} \mathrm{C}$ is attributed to quaternized graft chain degradation and the third stage of decomposition of the backbone polymer.

TGA thermograms, various kinetic parameters of degradation reaction have been determined by adopting most commonly used method of Horowitz-Metzger [22-27]. The activation energies of virgin and $\gamma$-irradiated samples of PEMA polymer corresponding with the major degradation process have been deducted using the expression [27].

$$
\ln \left(\ln \left[W_{0}-W_{f} / W_{t}-W_{f}\right]\right)=E_{a} \theta / R T_{s}^{2}
$$

where $W_{0}, W_{f}$ are the initial and final weights, $W_{t}$ is the remaining weight at temperature $T, E_{a}$ is the activation energy, $R$ is gas constant $\left(R=8.314 \mathrm{~J} \cdot \mathrm{k}^{-1} \cdot \mathrm{mol}^{-1}\right)$, and $\theta=$ $T-T_{s}$ with $T_{s}$ as the reference temperature corresponding to $W_{t}-W_{f} / W_{0}-W_{f}=1 / e$. In the light of the Eq.1, the activation energy $E_{a}$ can be calculated from the slope of the linear fitted line between $\ln \left(\ln \left[W_{0}-W_{f} / W_{t}-W_{f}\right]\right)$ and $\theta$ as illustrated in Figure 3, for virgin and $\gamma$-irradiated samples at doses 10, 20, 40, 60 and $100 \mathrm{kGy}$. The values of activation energies so determined for pristine as well as $\gamma$-irradiated samples have been enlisted in Table 1. There is a clear cut increase in the values of activation energy with the increasing irradiation dose. Such an increase may be attributed to the initialization of chain scissioning, possible evaporation of volatile side groups resulting in significant reduction of packing density, reorganization of molecular arrangements etc. in the polymeric sample which signifies the decrease in thermal stability of the polymer [22,23,28].

\subsection{Optical Studies}

\subsubsection{The Optical Absorption Edge}

The study of the optical absorption and particularly the absorption edge is an useful method for the investigation of optically induced transitions and for the provision of information about the structure and energy gap in both crystalline and non-crystalline materials. The principle of this technique is that a photon with energies greater than the band gap energy will be absorbed. The absorption coefficient $\alpha(\omega)$ depends exponentially on the photon energy $h \omega$. The absorption edge in many disordered materials follows the Urbach (1953) rule [29] given by:

$$
\alpha(\omega)=\beta \exp \left(\frac{h \omega}{\Delta E}\right)
$$

where $\alpha(\omega)$ is the absorption coefficient at an angular

Table 1. The values of various activation energy $E_{a}$, initial and final weights $\left(w_{0}, w_{f}\right)$ and remaining weight at temperature $T$ $\left(w_{t}\right)$ for virgin and $\gamma$-irradiated PEMA films.

\begin{tabular}{ccccc}
\hline Dose kGy & $E_{a}$ & $w_{0}$ & $W_{f}$ & $W_{t}$ \\
\hline 0 & 19.412 & 2.01 & 0.06 & 0.907826 \\
10 & 19.684 & $\mathbf{1 . 9 1}$ & $\mathbf{0 . 0 4}$ & 0.853043 \\
20 & 20.5 & 1.78 & 0.05 & 0.802174 \\
40 & 21.221 & 1.39 & 0.04 & 0.626957 \\
100 & 21.996 & 1.53 & 0.03 & 0.682174 \\
\hline
\end{tabular}




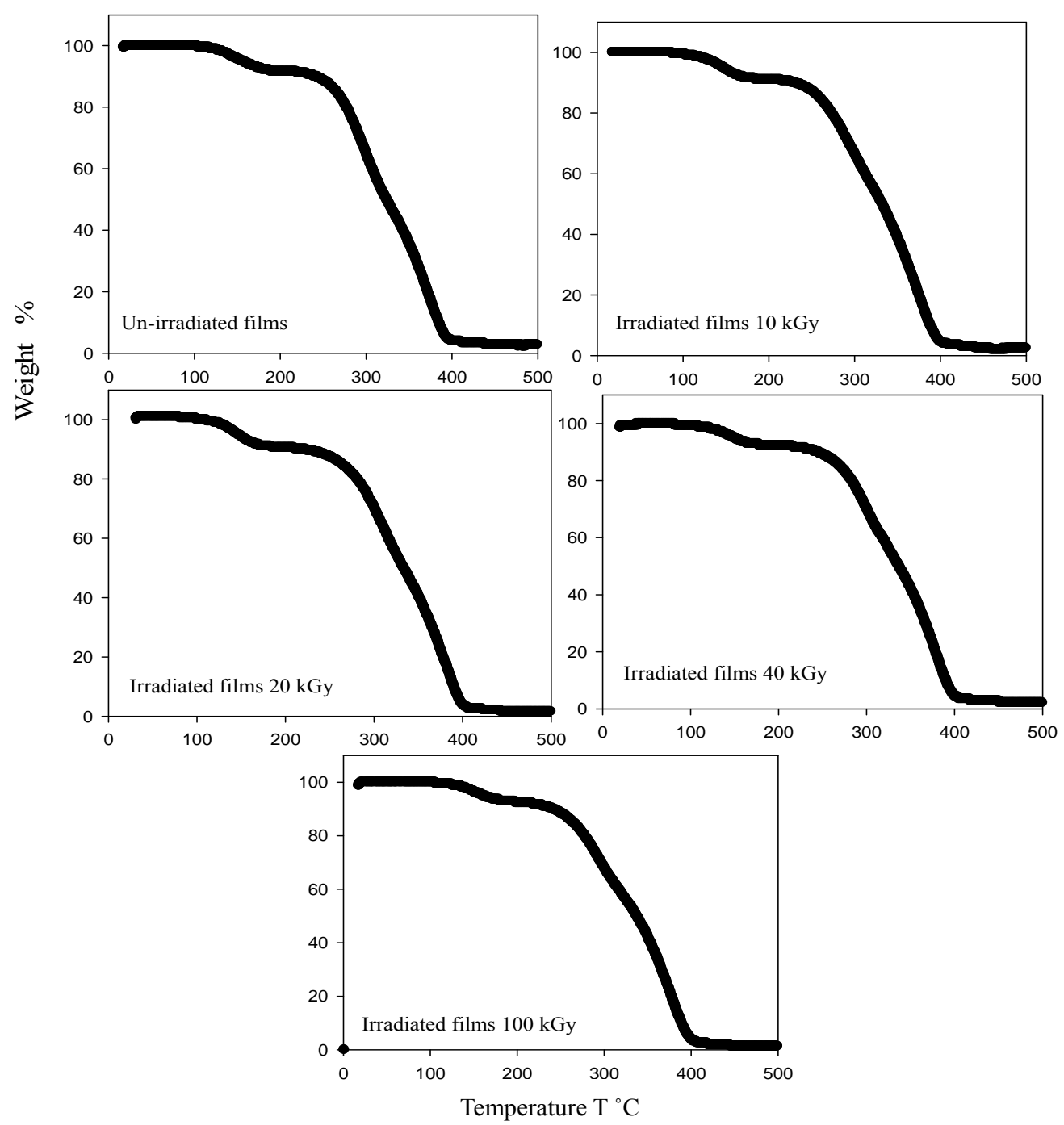

Figure 2. TGA thermograms for un-irradiated and irradiate PEMA films.

frequency of $\omega=2 \pi \nu$ and $\beta$ is a constant. $\Delta E$ is the width of tail of localized states in the band gap. The lower energy part of the absorption spectra gives information about the vibrations, and the higher part of the spectrum gives information about electronic states in the normal material. Figure 4 shows the absorption characteristic for the un-irradiated and irradiated EPMA Films. It is clear from Figure 4 that blank shows one absorption bands. The absorption at $325 \mathrm{~nm}$ is identified as the sorest band (B-band). These bands are attributed to the carbonyl group in polymeric macromolecule. It is observed that dose dependence UV has a slight effect on the position and the intensity of this band for PEMA films. Moreover, the intensity of carbonyl group peak decreases by increasing irradiation doses whereas its position is slightly shifted for all films.

\subsubsection{The Optical Band Gap}

A model based on the electronic transition between the localized states is not preferable. For higher values $\left(\alpha \geq 10^{4} \mathrm{~cm}^{-1}\right)$, the absorption coefficient (where absorption is associated with inter-band transitions) [30,31] takes the form:

$$
\alpha(\omega)=\beta\left(\hbar \omega-E_{\text {opt }}\right)^{M} / \hbar \omega
$$

$E_{\text {opt }}$ the optical gap, and a number that characterizes the transition process. The value of the constant $\beta$ in Eq.3 can be evaluated from the slope of the straight part in Figure 4. In the high absorption region where absorption is associated with inter-band transitions, there are two kinds of optical transition at the fundamental edge of crystalline and non-crystalline, direct transitions and indirect transitions. Both of which involve the interaction of an electromagnetic wave with an electron in valance band, which is then raised across the fundamental gap to the conduction band.

For indirect transitions, interactions with lattice vibrations (phonons) take place; thus the wave vector of the 


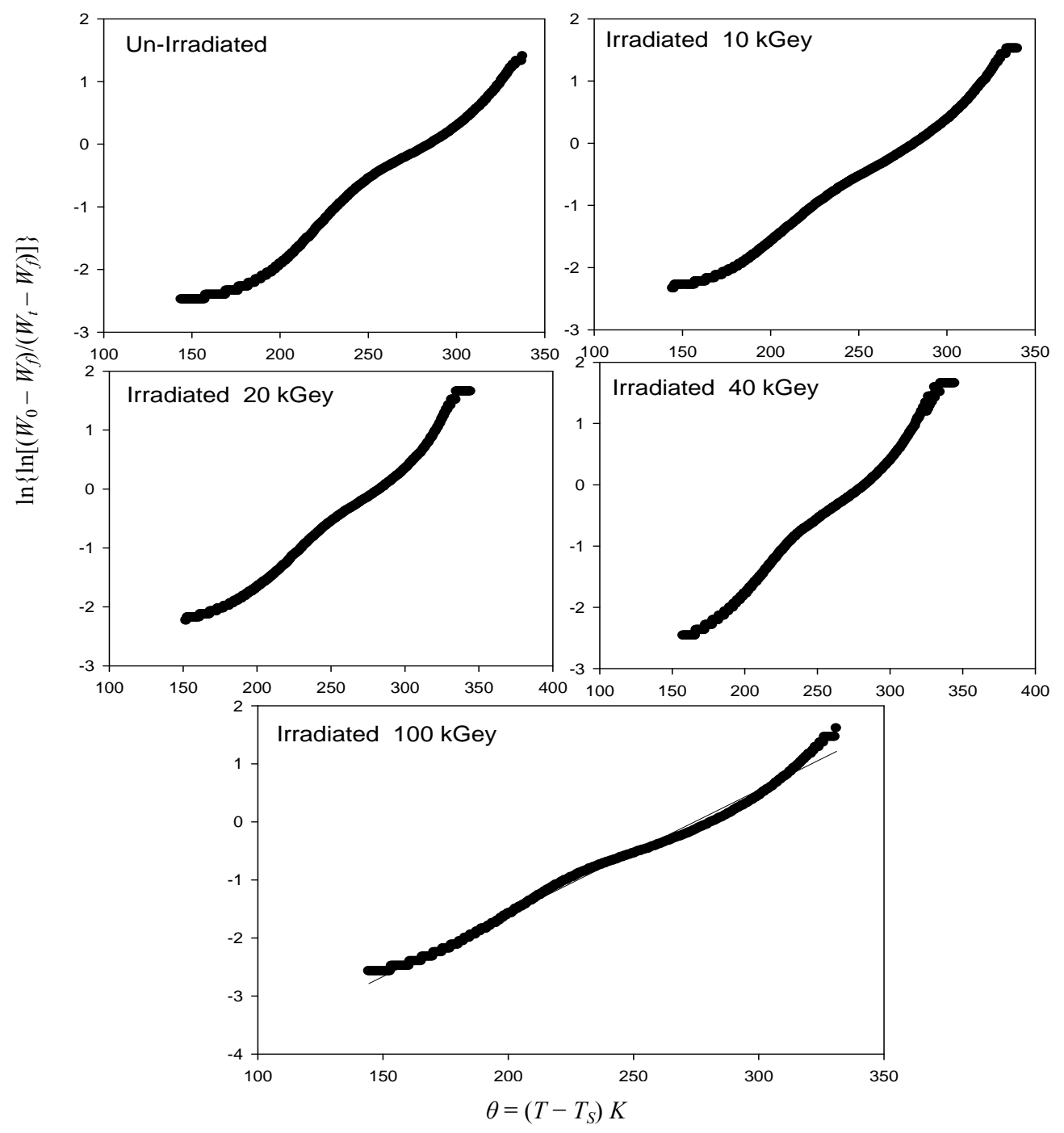

Figure 3. Plots of $\ln \left\{\ln \left(W_{0}-W_{f}\right) / I\right\}$ versus $q=\left(T-T_{s}\right) k$. For un-irradiated and irradiate PEMA films.

electron can change in the optical transition and momentum change will be taken or given up by phonons. In other words, if the minimum of the conduction band lies in a different part of K-space from the maximum of the valence band, a direct optical transition from the top of the valence band to the bottom of the conduction band is forbidden.

For direct transition $M=1 / 2$ or $3 / 2$ while for indirect transition $M=1,2$ and 3 which characterizes many amorphous or semi crystalline substances. The changes are strongly dependent on the internal structure of the absorbed substances. It may be expected that the gamma radiation can cause ionization or excitation of the optical electrons and possibly, displacement of atoms from their sites in the lattice of solid. When PEMA films are irradiated with radiation cross-linking and chain scission are formed. The optical band gap, $E_{\text {opt }}$ for un-irradiated and irradiated samples was determined using Eq.3, from the intercepts of the extrapolated linear part of plots of $(\alpha h v)^{1 / 2}$ versus $(h v)$, as shown in Figure 5. The values of $E_{\text {opt }}$ obtained are listed in Table 2 and it was found that $E_{\text {opt }}$ decreases with increasing of the irradiation dose.

\subsubsection{The Band Tail Width}

The band tail width was obtained using Eq.3 and then by plotting $(\ln \alpha)$ against $(h v)$ as shown in Figure 6. that should yield a straight line of a slope equals (1/ $\Delta E)$. It should mention that Eq.3 is applied only in the low absorption region. The band tail width $E_{e}$ is listed in Table 2, it increases with increasing dose; i.e. $E_{e}$ exhibits an opposite trend with $E_{\text {opt }}$. This is could be due to the fact that, when polymer exposed to irradiation doses, it starts to crosslink, a formation of new covalent bonds can be produced. Then, different chains are obtained, i.e. cross 


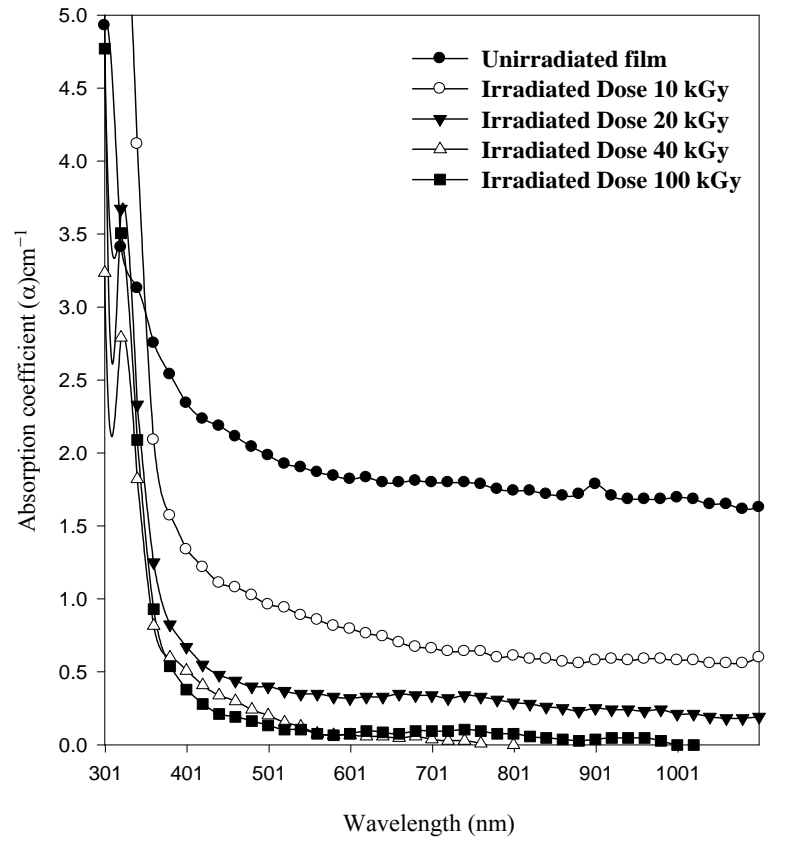

Figure 4. Effect of the irradiation gamma doses on the absorption coefficient $(\alpha)$ with wavelength for PEMA films.

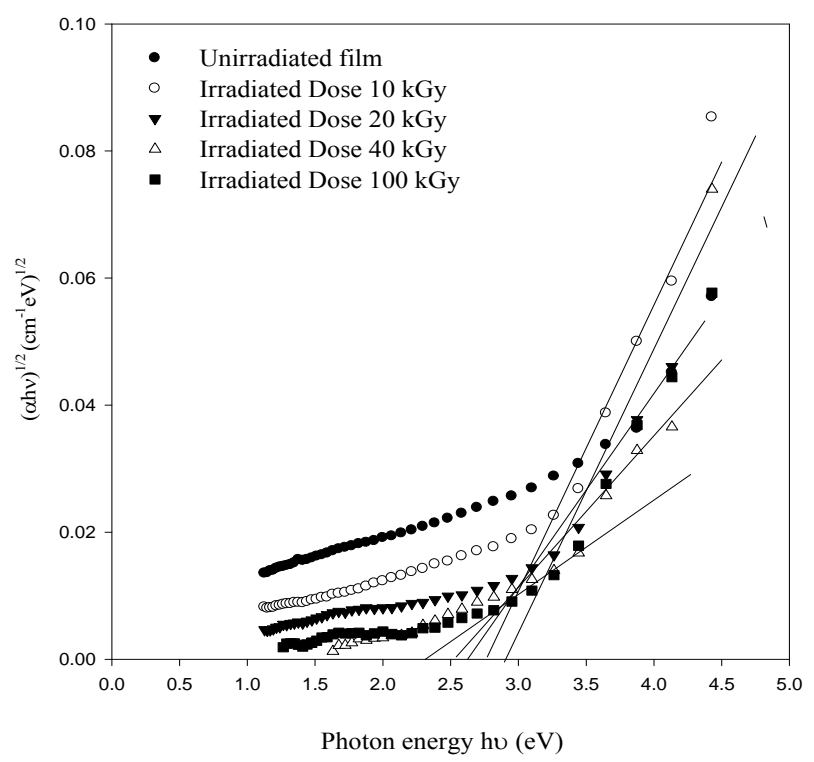

Figure 5. The optical absorption coefficient edge plotted as $(\alpha h v)^{1 / 2}$ versus photon energy $(h v)$ for as deposited and irradiated PEMA films with different doses.

linking starts which are in turn hinder the motion of molecules and reduce its activities and in turn decrease the optical band gap.

On further gamma doses, a formation of free radicals are obtained, which are causes a decrease in the optical band gap. As the gamma dose increases from 10 to 100 $\mathrm{kGy}$, the crystalline structure is assumed to be perturbed and leading to an increase in the degree of disorder. At

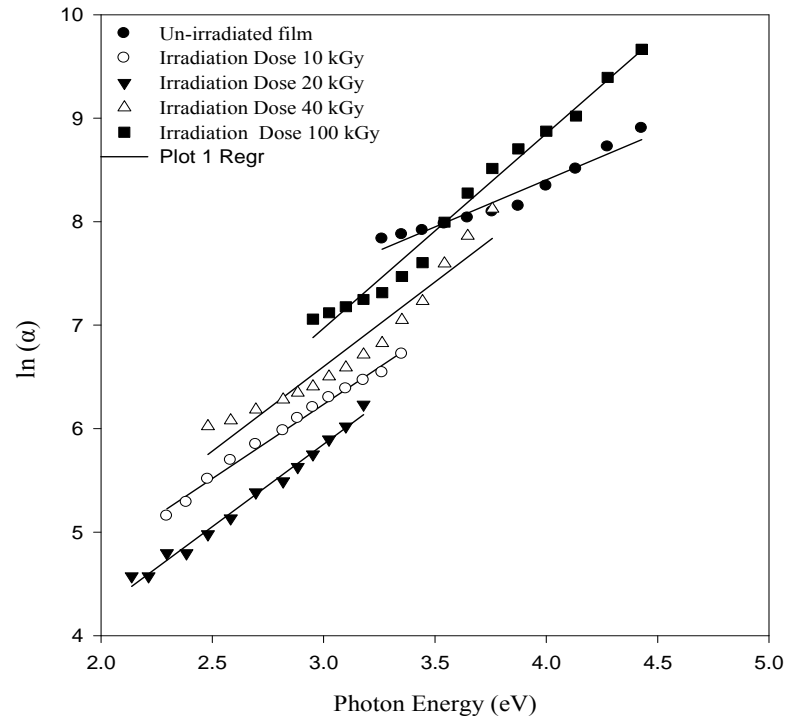

Figure 6. The logarithmic variation of absorption coefficient $\ln (\alpha)$ versus photon energy for PEMA films irradiated with different doses.

Table 2. Optical parameters for PEMA films under different $\gamma$ irradiation doses.

\begin{tabular}{ccc}
\hline PEMA Films & $\begin{array}{c}\text { Optical band } \\
\text { gap (eV) }\end{array}$ & $\begin{array}{c}\text { Band tail } \\
\text { width (eV) }\end{array}$ \\
\hline Un-irradiated films & 2.95 & 0.53 \\
Irradiation dose $10 \mathrm{kGy}$ & 2.80 & 0.61 \\
Irradiation dose $\mathbf{2 0} \mathbf{~ k G y}$ & 2.65 & 0.63 \\
Irradiation dose $\mathbf{4 0} \mathbf{~ k G y}$ & 2.55 & 0.70 \\
Irradiation dose $\mathbf{1 0 0} \mathbf{~ k G y}$ & 2.45 & 1.1 \\
\hline
\end{tabular}

higher dose (100 kGy), the chains scission become predominant relative to the crosslinking. From the density of states model it is known that $E_{\text {opt }}$ decreases with increasing the degree of disorder of amorphous phase. Furthermore, the band tailing shifts to higher energies as shown in Figure 5 and extends into the forbidden band.

\subsubsection{Dispersion Analysis}

In this work, the polymer films are sufficiently thick compared to the beam wavelength to neglect the interference phenomena. The air/polymer interface transmission $T$ are expressed as functions of refractive index $(n)$ and extinction coefficient $(K)$ [32].

$$
T=\frac{4 n}{(n+1)^{2}+K^{2}}
$$

Once the measurements $T$ have been performed, an iterative procedure to calculate $n$ and $K$ is initiated. When the absorption $\alpha$ is known, the extinction coefficient $K$ can be found the relation $K=\alpha \lambda / 4 \pi$. The real and 
imaginary parts of dielectric constant and can be estimated if the refractive index and extinction coefficient is known from the relations $\varepsilon^{\prime}=\left(n^{2}-K^{2}\right)$ and $\varepsilon^{\prime \prime}=2 n K$. The real part generally relates to dispersion, while the imaginary part provides a measure to the dissipative rate of the wave in medium [33].

The refractive index and the single oscillation parameters have been calculated and discussed in terms of the Wemple-DiDmenico model [34] Figure 7. That the parameters single-oscillation energy $\left(E_{0}\right)$ and dispersion energy $\left(E_{d}\right)$ have been calculated by knowing the refractive index $(n)$ which is independent on the scale of optical dielectric constant $(\varepsilon)$ and is consequently an "average" energy gap, where as $E_{d}$ is depends on the scale of $\varepsilon$ and thus serves as an interband strength parameters. Since the $M_{o}$ and $M_{d}$ moments are involved most heavily near the interband absorption edge [35].

The dispersion of refractive index below the interband absorption edge according to the single oscillator model is given by [34].

$$
n^{2}(E)=1+\left[\frac{E_{0} E_{d}}{E_{0}^{2}-E^{2}}\right]
$$

where the parameters $E_{o}, E_{d}$ are the single-oscillation energy and dispersion energy respectively. By plotting $\left(n^{2}-1\right)^{-1}$ versus $E^{2}$ and fitting a straight line as shown in Figure 8; $E_{0}$ and $E_{d}$ are determined directly from the gradient, $\left(E_{0}, E_{d}\right)^{-1}$ and the intercept $\left(E_{0} / E_{d}\right)$, on the vertical axis, Table 3 and Figure 9 [35-37].

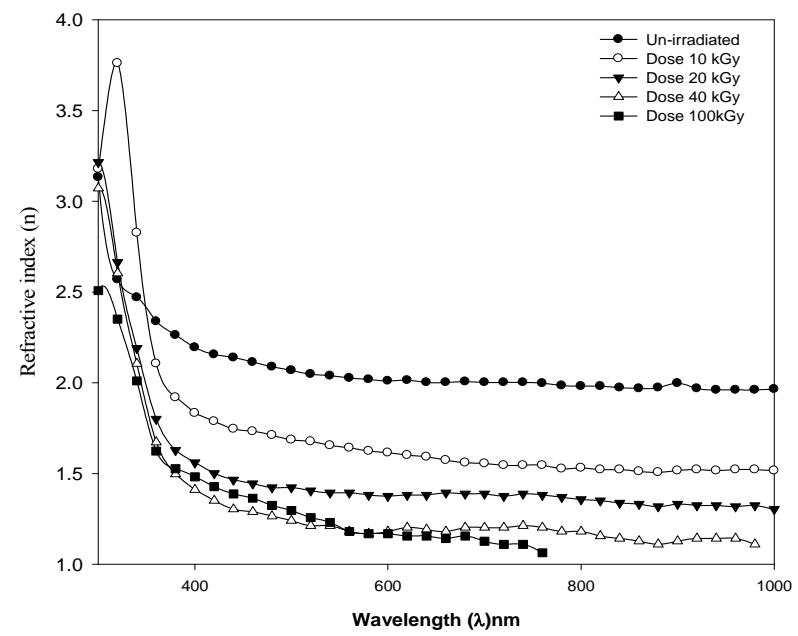

Figure 7. Refractive index $(n)$ against wavelength $(\lambda) \mathrm{nm}$, for PEMA film before and after irradiation with different dose.

\section{CONCLUSIONS}

From the results and discussion above it is possible to conclude that:

1) XRD obtained for PEMA films confirmed the amorphous nature and partially crystalline nature for different

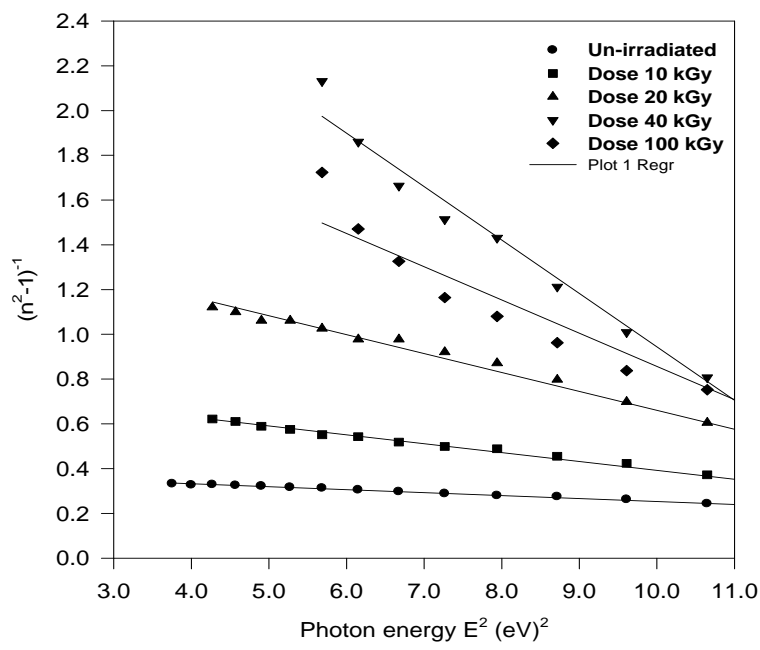

Figure 8. Plot of refractive index factor $\left(n^{2}-1\right)^{-1}$ versus $E^{2}$ for un-irradiated and irradiated PEMA films.

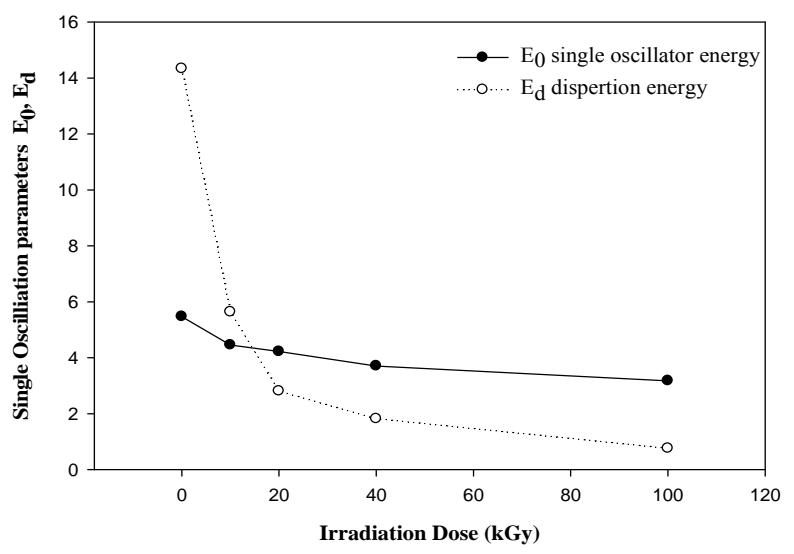

Figure 9. The variation of $E_{0}$ single oscillator energy and $E_{d}$ dispersion energy with gamma irradiation dosed $f$ or PEMA films.

Table 3. The calculated single-oscillation parameters values of PEMA films.

\begin{tabular}{ccc}
\hline Gamma dose & $\boldsymbol{E}_{\mathbf{0}}$ & $\boldsymbol{E}_{\boldsymbol{d}}$ \\
\hline Un-irradiated & 5.083256 & 12.65121 \\
$\mathbf{1 0} \mathbf{~ k G y}$ & 4.4832 & 5.657539 \\
$\mathbf{2 0} \mathbf{~ G G y}$ & 4.22017 & 2.802622 \\
$\mathbf{4 0} \mathbf{~ G y}$ & 3.803229 & 1.220061 \\
$\mathbf{1 0 0} \mathbf{~ G G y}$ & 3.598682 & 0.983112 \\
\hline
\end{tabular}

films. The XRD data suggested that the crosslinking process predominated during the irradiation of PEMA.

2) TGA studies revealed that the thermal stability of polyethyl methacrylate.

3) Gamma-rays irradiation of PEMA films in the dose range of 10 up to $100 \mathrm{kGy}$ has been investigated as a potential technique of refractive index modulating opti- 
cal elements. There was a decrease in the refractive index in via the decrease in the dispersion energy for the irradiated films with different doses.

4) Single oscillator energies in the PEMA films were significantly influenced by gamma irradiation affects. It was observed a decrease in the dispersion energy and discussed in terms of the Wemple-DiDmenico model.

5) The affect of gamma irradiation on the optical band gap obtained from. Moreover, there was a decrease in the optical energy gap with increasing radiation dose up to $100 \mathrm{kGy}$. It was deduced that the energy of gamma rays, in the dose range from 10 up to $100 \mathrm{kGy}$, is suitable for the cross-linking of PEMA films.

\section{REFERENCES}

[1] Paul, S. (1996) Surface coatings science and technology. Wiley, New York, 312.

[2] Odian, G. (1991) Principles of polymerization. John Wiley \& Sons Inc., New York, 311-313.

[3] Shetter, J.A. (1963) Effect of stereoregularity on the glass temperatures of a series of polyacrylates and polymethacrylates. Journal of Polymer Science, B, 1, 209-213. doi:10.1002/pol.1963.110010503

[4] Hata, T., Nose, T. and Polym, J. (1967) Stress-strain-time relation of poly(n-alkyl methacrylates) in rubbery state. Science, C, 16, 2019.

[5] Gardlund, Z.G., Laverty, J.J. and Polym, J. (1969) Polyalkylmethacrylate films as matrices for photochromic studies. Science, B, 7, 719.

[6] Grassie, N., Mc Callum, J.R. and Polym, J. (1964) Thermal and photochemical degradation of poly(n-butyl methacrylate. Science, A, 2, 983.

[7] Smith, S.D., Long, T.E. Mc Grath, J.E. and Polym, J. (1994) Thermogravimetric analysis of poly(alkyl methacrylates) and poly(methylmethacrylate- $g$-dimethyl siloxane) graft copolymers. Science-Chemistry A, 32, 1747.

[8] Malhotra, S.L., Minh, L., Blanchard, L.P. and Macromol, J. (1983) Thermal decomposition and glass transition temperature of poly-(phenylmethacrylate) and poly (cyclohexyl methacrylate). Science-Chemistry A, 19, 559.

[9] Ahmed, M.T. (2010) Relaxational study of poly(ethylmthacrylate) by thermally stimulated depolarization current, thermal sampling spectroscopy: Modified dipoledipole interaction theory. Journal of the Korean Physical Society, 57, 272-281. doi:10.3938/jkps.57.272

[10] Maitra, S., Bandyopadhyay, N., Das, S., Pal, A.J. and Pramanik, J. (2007) Non-isothermal decomposition kinetics of alkaline earth metal carbonate. Journal of the American Ceramic Society, 90, 1299-1303. doi:10.1111/j.1551-2916.2007.01607.x

[11] Friedman, H.L. (1964). Kinetic of thermal degradation of char-forming plastics from thermogravometry. Application to a phenolic plastic. Journal of Polymer Science Polymer Symposium, 6, 183-195. doi:10.1002/polc. 5070060121
[12] Nam, J.D. and Seferis, J.C. (1991) Composite methodology for multistage degradation of polymer. Journal of Polymer Science: Part B, Polymer Physics, 29, 601-608. doi:10.1002/polb.1991.090290509

[13] Anderson, D.A. and Freeman, E.S. (1961) The kinetics of thermal degradation of polyethylene and polystyrene. Journal of Polymer Science, 54, 253-260. doi:10.1002/pol.1961.1205415920

[14] Radhakrishnan, T.S. (1999) New method for evaluation of kinetic parameters and mechanism of degration from pyrolysis - GC studies: Thermal degradation of polydimethylsiloxanes. Journal of Applied Polymer Science, 73, 441-450.

doi:10.1002/(SICI)1097-4628(19990718)73:3<441::AIDAPP16>3.0.CO;2-J

[15] Fink, D., Ed. (2004) Fundamentals of ion-irradiated polymers. Springer-Verlag, Berlin, 7-84.

[16] Sharma, T., Aggarwal, S., Kumar, S., Mittal, V.K., Kalsi, P.C. and Manchanda, V.K. (2007) Effect of gamma irradiation on the optical properties of CR-39 polymer. Journal of Materials Science, 42, 1127-1130. doi:10.1007/s10853-006-0516-7

[17] Abiona, A.A. and Osinkolu A.G. (2010) Gamma-irradiation induced property modification of polypropylene. International Journal of Physical Science, 5, 960-967.

[18] Zaki, M.F. (2008) Gamma-induced modification on optical band gap of CR-39 SSNTD. Brazilian Journal of Physics, 38, 558-562. doi:10.1590/S0103-97332008000500005

[19] Fink, D., Chung, W.H., Klett, R., Schmoldt, A., Cardoso, J., Montiel, R., Vazquez, M.H., Wang, L., Hosoi, F., Omichi, H. and Goppelt-Langer, P. (1995) Carbonaceous Clusters in irradiated polymers as revealed by UV-Vis spectrometry. Radiation Effects and Defects in Solids, 133, 193-208. doi:10.1080/10420159508223990

[20] Nair, C.P.R., Bindu, R.L. and Joseph, V.C. (1995) Cyanate esters based on cardanol modified-phenol-formaldehyde resins: Syntheses and thermal characteristics. Journal of Polymer Science, Part A: Polymer Chemistry, 33, 621. doi:10.1002/pola.1995.080330403

[21] Tang, W., Li, X.G. and Yan, D. (2004) Thermal decomposition kinetics of thermotropic copolyesters made from trans-p-hydroxycinnamic acid and p-hydroxybenzoic acid. Journal of Applied Polymer Science, 91, 445-454. doi:10.1002/app.13103

[22] Nouh, S.A., Atta, M.R. and El-Melleegy Rad, W.M. (2004) A study of the effects of gamma and laser irradiation on the thermal, optical and structural properties of CR-39 nuclear track detector. Radiation Effects and Defects in Solids, 159, 461-474. doi:10.1080/10420150412331296835

[23] Kalsi, P.C., Mudher, K.D.S., Pandey, A.K. and Iyer, R.H. (1995) Thermal studies on unirradiated and $\gamma$-irradiated polymer of allyl diglycol carbonate. Thermochimica Acta, 254, 331-336. doi:10.1016/0040-6031(94)01989-T

[24] Mallikarjun, K.G. (2004) Thermal decomposition kinetics of $\mathrm{Ni}(\mathrm{II})$ chelates of substituted chalcones. E-Journal of Chemistry, 1, 105-109. doi:10.1155/2004/385034 
[25] Jellinek, H.H.G. (1978) Aspects of degradation and stabilization of polymers. Elsevier Scientific Publishing Company, New York.

[26] Singh, B.K., Kumari, P., Prakash, A. and Adhikari, D. (2009) Thermal decomposition kinetics of peanut shell. Nature and Science, 7, 73-78.

[27] Horowitz, H.H. and Metzger G. (1963) A new analysis of thermogravimetric traces. Analytical Chemistry, 35, 14641468. doi:10.1021/ac60203a013

[28] Anslyn, E.V. and Dougherty, D.A. (2006) Modern Physical organic chemistry. Edwards Brothers, Inc., Violet Hill.

[29] Urbach, F. (1953) The long-wavelength edge of photographic sensitivity and of the electronic absorption of solids. Journal of Physical Reviews, 92, 1324. doi:10.1103/PhysRev.92.1324

[30] Ilyas, M., Zulfequar, M. and Husain, M. (2000) Optical properties of a- $\left(\mathrm{Se}_{70} \mathrm{Te}_{30}\right)_{100-\mathrm{x}}\left(\mathrm{Se}_{98} \mathrm{Bi}_{2}\right)_{\mathrm{x}}$ thin films. Journal of Modern Optics, 47, 663.

[31] Mohamed, S.H., Ahmed, A.M., Diab, A.K. and Wakkad, M.M. (1999) Structural and optical properties of Ge-AsTe thin films. The European Physical Applied Journal, 8, 215-224.

[32] Soldera, A. and Monterrat, E. (2002) Mid-infrared optical properties of a polymer film: Comparison between classical molecular simulations, spectrometry, and ellipsometry techniques. Polymer, 43, 6027-6035.

doi:10.1016/S0032-3861(02)00467-6

[33] Rajesh, K.R. and Menon, C.S. (2002) Estimation of the refractive index and dielectric constants of magnesium phthalocyanine thin films from its optical studies. Materials Letters, 53, 329-332. doi:10.1016/S0167-577X(01)00502-X

[34] Wemple, S.H. and DiDmenico, W. (1971) Behavior of the electronic dielectric constant in covalent and ionic materials. Physical Review B, 3, 1338-1351. doi:10.1103/PhysRevB.3.1338

[35] Dixon, J.R. and Riedl, H.R. (1965) Optical dispersion of lead sulfide in the infrared. Journal of Physical Review A, 140, 1283-1291. doi:10.1103/PhysRev.140.A1283

[36] Sokkar, T.Z.N., El-Farahary, K.A. and El-Bakary M.A. (2003) Determination of optical properties, dispersion, and structural parameters of poly(ethylene terephthalate) fibers using automatic variable wavelength interferometry technique. Journal of Applied Polymer Science, 89, 1737. doi:10.1002/app.12189

[37] Abul-Hail, R.Ch. (2010) Optical absorption of polycarbonate (Makrofol E) as means of gamma-ray dosimetry. Journal of Basrah Researches (Sciences), 36, 26. 